\title{
The Predictive Role of Resilience in Psychological Immunity: A Theoretical Review
}

\section{IJCRR \\ Section: Healthcare \\ Sci. Journal Impact \\ Factor: 6.1 (2018) \\ ICV: 90.90 (2018) \\ (c) (i) (8) \\ Copyright@IJCRR}

\section{ABSTRACT}

Background: Psychological immunity comprises an individual's ability to safeguard and promote mental health. Oláh's psychological immune system is a multi-dimensional model which incorporates factors affecting mental health like optimism, selfesteem, and control over emotional faculties; it consists of personal protective and promotive resources that offer immunity to environmental stress. This model can be useful for exploring relevant psychological phenomena like resilience and the cognitive and behavioural concepts associated with it.

Objective: To describe psychological immunity and the key concepts driving it.

Method: Narrative review of literature relevant to psychological immunity.

Conclusion: It has been pointed out that the factors influencing resilience have useful equivalents within the psychological immune system model, implying that resilience is a key factor promoting psychological immunity. The psychological immune system model will be important for investigating interactions with the biological immune system, especially concerning interventions that can enhance health, like resilience training. Furthermore, the model can act as a unifying framework for psychological phenomena like well-being and coping.

Key Words: Psychological immunity, Resilience, Health Psychology, Covid impact

\section{INTRODUCTION}

Individuals often experience several challenges during their lives, ranging from everyday difficulties to major crises. All manner of personal, professional, and financial problems are on the rise, resulting in the degradation of mental wellness. However, people experience these problems differently, which determines the degree of trauma that occurs. For instance, some people are liable to be overwhelmed by day to day tasks, while other individuals perceive potentially traumatic events as merely challenging. ${ }^{1}$ This variance in the perception of stress or pressure must be investigated and understood.

Concepts like resilience and psychological immunity help expand research into mental health beyond medical and psychological risk factors, extending inquiry into wellness research like how the properties of infection-resistant groups are studied during an epidemic. ${ }^{2}$ Earlier, the term 'resilience' involved a negative approach of absence in psychopathology. ${ }^{3}$ An eventual shift in psychological thought has transformed the concept of resilience into a construct involving mental health risk factors and the identification of individual characteristics that beget higher competence, adaptive behaviour, and resistance to stress and psychological illnesses. ${ }^{1,3}$ Resilience has a strong influence on mental health; from inference, it can be understood that it has relevance in other contexts like education, policymaking, business, and public welfare. Hence, the factors that contribute to the development of resilience have to be studied carefully. Unfortunately, researching resilience proves difficult because of the many inconsistencies in how the concept is defined and operationalized. For instance, resilience has been defined by researchers as a process, trait, or outcome. Such variations require the concept to be delimited to determine the nature and vector of research inquiry. Conceptual delimitation will help better evaluate and compare the results of resilience research, making it easier to analyse and operationalize. ${ }^{1}$ This is where the concept of psychological immunity comes into play.

\section{Corresponding Author:}

Tanveer Kaur, Amity Institute of Psychology and Allied Sciences, AUUP, Noida, Uttar Pradesh 201313, India.

ISSN: 2231-2196 (Print)

Received: 10.07 .2020
ISSN: $0975-5241$ (Online)

Revised: 11.09 .2020
Accepted: 18.10 .2020 
The psychological immune system (PIS) - which brings about psychological immunity - is a construct that shares some similarities with the biological immune system. ${ }^{4}$ For instance, they both include autonomous and adaptive mechanisms that protect and self-heal; implicit processes like habituation and emotional processing in the PIS are similar to the adaptive immunity process of the BIS in that they promote healing, are automatic, and operate outside of human awareness. ${ }^{4}$ Similarly, both these systems can be primed using interventions. However, one major difference between the PIS and the BIS is that the former involves two parallel processes: an automatic and silent one similar to the BIS, and a proactive interventionist process. ${ }^{4}$ Furthermore, cognitive interventions are at the core of many interventions related to the psychological immune system. The competencies associated with psychological immunity aid in promoting resilience and fighting off stress. ${ }^{5}$ The construct of the psychological immune system can help collate a lot of established concepts into a single framework. For example, research into emotional processing, wellbeing, and resilience can be folded into the umbrella of psychological immunity research. This will help reasonably decrease the number of inconsistencies that arise from the various conceptualizations of resilience and other psychological concepts related to it. Furthermore, psychological immunity serves to encompass both protective and promotive psychological mechanisms within a cognitive framework in a manner that does not simply denote resilience as a catch-all term for both kinds of processes. This is in line with the thinking of several researchers like George Bonanno, who argued that there has to be a clear delineation between the definitions of resilience - a protective process - and coping, a healing process. ${ }^{6} \mathrm{With}$ these ideas in mind, the first section of this paper will elaborate upon the PIS. The next section will elaborate upon the various definitions of resilience and the theories associated with it. Finally, the integration of resilience within the psychological immunity framework will be discussed.

\section{Psychological Immunity: Concepts and Theory}

Psychological immunity has been defined as a complex mechanism that runs in parallel with biological immunity. ${ }^{7}$ Its primary functions are said to be the recognition, projection, and management of stressful situations to maintain the integrity of one's personality when interacting with the external environment. ${ }^{7}$ Psychological immunity helps develop appropriate behaviour and boost adaption to changing circumstances. The proactive and promotive mechanisms involved in psychological immunity help enhance an individual's well-being. ${ }^{8}$ Despite psychological immunity having been identified as a safeguard that prevents various situational risks, there are very few studies that investigate the construct. Oláh's psychological immune system is one synthesized framework that incorporates many characteristics that are related to psychological immunity. This construct also has the potential to act as a framework for several established phenomena such as well-being, emo- tional processing, and resilience. ${ }^{9}$ Oláh's model elaborates the cognitive influences that involve an individual's interpersonal and intrapersonal appraisals, their execution of behaviour, and the evaluations of such behaviour. ${ }^{5}$

Oláh's model of the psychological immune system describes an integrated construct composed of 16 promotive and protective personal competencies that help manage environmental stress. ${ }^{5}$ These personal competencies are multi-dimensional, incorporating cognitive, behavioural, and trait-like factors. This model operates as a superordinate system composed of three subsystems in constant interactions. These subsystems are:

- Approach-belief subsystem

- Monitoring-creating-executing subsystem

- Self-regulating subsystem

The first, the Approach-belief subsystem (ABS), is supposed to help people adjust to their environment. It helps individuals appraise scenarios and distinguish them into a spectrum of meaningful and potentially threatening. The six components of this subsystem are devised as positive definitions of the self, with the individual having the potential to be competent and goal-oriented. ${ }^{5}$ The ABS contains the operational factors of:

- Optimism

- Coherence feeling

- Development feeling

- Control ability

- Personal source monitoring ability

- Social source monitoring ability ${ }^{10}$

Optimism involves beliefs that current events are proceeding towards ideal situations. Coherence feeling involves the harmonization between thoughts, emotions, behaviour, and lifestyle. Development feeling involves a continual experience of self-development and achievement. Control ability implies the capacity to control one's own emotion. ${ }^{10}$ Personal source monitoring ability implies one's openness to novel phenomena and development, while Social source monitoring ability indicates the sensitive capacity to selectively observe and use socio-environmental cues and information. The ABS focuses on an approach orientation, reinforcing the anticipation of positive outcomes, belief in the comprehensibility and manageability of the environment, and motivation for self-actualization. ${ }^{5}$

The second subsystem of Oláh's model is the Monitoringcreating-executing subsystem (MCES). ${ }^{5}$ The five components of MCES help prompt the exploration of physical, social, and interpersonal phenomena for finding new challenges and experiences. The MCES includes:

- Personal source mobilizing ability

- Personal source creating ability

- Social source mobilizing ability 
- Social source creating ability

- Self-respect

Personal source mobilizing ability involves mechanisms for strengthening belief, achieving goals, and selecting appropriate behaviour. ${ }^{10}$ Personal source creating ability implies an individual's degree of inventiveness, ingenuity, or creativity in developing, managing, and restructuring life plans and information based on personal realisations. Social source mobilizing ability involves an individual's capacity to make connections, gain social capital, and obtain consensus. Social source creating ability involves the mechanisms that help build and organize teams and collaboration. ${ }^{10}$ Finally, Self-respect involves the degree of positive and realistic selfestimation, self-esteem, and temperance when it comes to pride. In addition to the actualization of factors like creative self-concept and social mobilization capacity, the components of the MCES involve the innovative capacity to generate new ideas and alternative possibilities, all of which are important for personal and social adaptation. ${ }^{5}$

The third and last subsystem within Oláh's model is the selfregulating subsystem (SRS), which stabilizes the ABS and the MCES by managing tenuous emotions and thoughts that interfere with planned behaviour. The five components of this system include:

- Emotion control

- Excitability control

- Impulsivity control

- Persistence

- Synchrony ability ${ }^{10}$

Emotional control implies an individual's ability to constructively transform negative emotions. Excitability control involves the capacity to control anger and constructively apply it. Impulsivity control is the ability to control personal notions, rationalize positive behaviour, and select appropriate behaviour within context. ${ }^{10}$ Persistence denotes the ability to accomplish tasks despite being hindered by obstacles; it also implies a high tolerance for frustration. Synchrony ability involves the capacity to perceive environmental changes while attending to personal activities.

The 3 aforementioned subsystems form a multi-dimensional construct that offers immunity against stress and psychological trauma by continually exploring the transient environment and assimilating unique experiences. ${ }^{11}$ Furthermore, this process also occurs within the individual's psyche as well. The synchronization of such exploration and assimilation orients an individual towards their environment. Therefore, the psychological immune system creates a balance between personality functions and environmental factors to increase resilience and mental fitness. ${ }^{11}$

Oláh's psychological immune system is relevant to both mental and physical illnesses. For instance, positive and negative cognitive appraisals of difficulties/trauma/danger can promote behaviours that stabilise or destabilise the psychological immune system respectively. ${ }^{4}$ For instance, cognitive influences determine the choice to seek medical attention, while negative appraisals can affect mental stability can cause episodes of fear or panic. Hence, the psychological immune system can help codify changes in health behaviour, accounting for relevant psychological phenomena like emotional processing, well-being, and resilience.

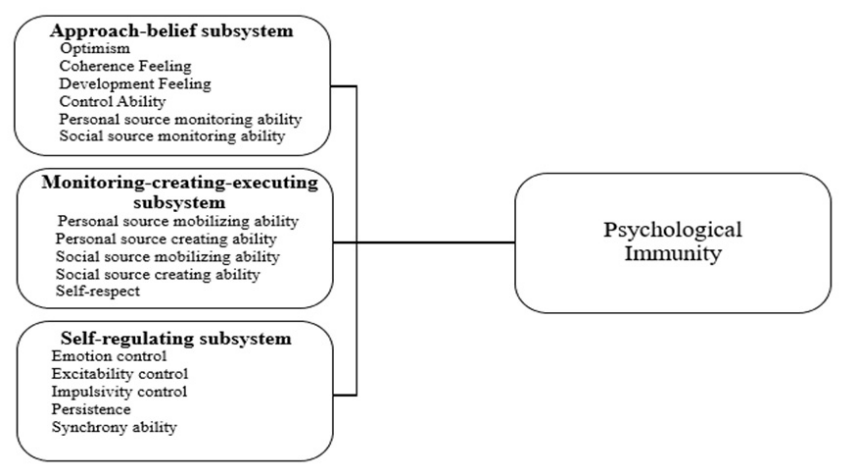

Figure 1: Antecedents of Psychological Immunity according to Oláh's psychological immune system model.

\section{Implications on Resilience}

There have been numerous debates on how resilience should be defined and conceptualized, with many researchers analysing and elaborating upon its essential attributes. It has been established that the primary driving for resilience in adversity, and its effect is positive adaptation. ${ }^{1}$ Since resilience is a combination of several factors, the development of its conceptual limitations is a responsibility in many disciplines like medicine, psychology, pedagogy, social work, etc. ${ }^{2}$ This has resulted in numerous definitions and conceptualizations for resilience. For instance, while resilience is said to result in good psychological outcomes despite high-risk experiences, researchers opine that it can also help individuals develop in difficult circumstances. ${ }^{3}$ The concept has also been defined as decreased vulnerability across emotional, cognitive, and social capacities. ${ }^{3}$ Furthermore, there is some consternation as to whether resilience should be conceptualized as a trait or process. ${ }^{1}$ As a trait, resilience can be conceptualized as being a combination of factors like optimism, curiosity, selfefficacy, self-esteem, and the ability to detach and rationalize problems. These factors act as protective characteristics that influence and change individual responses to high-risk situations that have the potential to result in maladaptive outcomes. ${ }^{1}$ However, researchers have identified that resilience involves both protective and promotive factors, with the latter implying that future experiences will result in more success. Given this distinction, it is difficult to conceptualize resilience as a trait since it could then be construed as binary 
i.e. something one has or does not have. Hence, the factors behind resilience should take into account their relation to specific functions, the environment, and other factors. ${ }^{1}$ This means that the psychological immune system will work as a better framework for developing future research on psychological resilience.

When resilience is conceptualized as a process - as many researchers have in the past - effects of the factors behind it still vary contextually and temporally. ${ }^{1}$ This means that individuals who respond positively to adverse, stress-inducing events need not react the same way in the future, or in different contexts. Resilience research on athletes has reported that positive adaptations are accumulative and involve several shifts in thoughts. ${ }^{1}$ Hence, it can be understood that resilience could even decline as a result of intrapersonal or environmental factors. The multi-dimensional nature of resilience is exactly what has resulted in the debate over its conceptualization. By folding resilience into the framework of the psychological immune system, we account for many intrapersonal and environmental factors that result in resiliency. Additionally, this allows resilience to be viewed in the form of a loose gradient rather than a binary trait.

Regardless of the confusion behind its conceptualization, the aspects of resilience are fairly clear. An individual's resilience is determined by their ability to achieve positive results in highly risky situations, their ability to function well when affected by acute or chronic stress, and the ability to recover from resultant trauma. ${ }^{3}$ In line with these claims, resilience involves characteristics like control over the memory of traumatic incidents, integration of emotions and memories, regulation of emotions and symptoms, self-esteem, internal cohesion, understanding of traumatic impact, and ability to develop positive meaning. In light of these characteristics, Truffino suggested that resilience involves a state of emotional and social competence that is oriented towards the future. ${ }^{3}$ By his definition, social competence implies the capacity to form optimal social relationships, while emotional competence involves a positive self-conception, self-control and emotional regulation, and personal autonomy. Several of the aspects and characteristics of resilience have analogues in the psychological immune system, which implies that it could be a useful framework for guiding research into resilience and other similar phenomena like coping. The aforementioned framework for the psychological immune system delineates between the phenomena of resilience and coping since resilience modifies how situations are appraised while coping involves strategies that are employed after situations are appraised. ${ }^{1}$ For instance, components of the MCES like personal source mobilizing ability, personal source creating ability, social source mobilizing ability, and social source creating ability help select for coping strategies, while components of the ABS and SRS like Coherence feeling, Development feeling, and Control ability, and Emotional control are factors closely related to the characteristics of resilience.

\section{Perspective}

The psychological immune system represents the idea that the biological immune system has a psychological counterpart. The psychological immune system focuses on interpersonal and environmental effects on the individual; it helps form a better picture of how cognitive influences affect physical and mental health. The psychological immune system can help encompass the construct of wellbeing, which is an important topic in positive psychology. ${ }^{4}$ Elements of well-being include achievements, personal engagement and meaning, positive emotions, and relationships. As such, well-being has defensive characteristics when it comes to physical health. For instance, a long-term study opined that positive emotions and wellbeing results in less risk of mortality, natural or otherwise. ${ }^{4}$ Similarly, there is a proven link between life-expectancy and psychological immune competency. ${ }^{12}$ Optimism - a key subcomponent of the ABS - is a significant predictor of positive physical health, higher resilience, quality of life, and better psychological well-being. ${ }^{4,8}$ These correlations indicate that the psychological immune system can incorporate psychological phenomena like wellbeing and resilience.

The psychological immune system can help incorporate various pieces of health-related evidence not expanded upon in the biological immune system. ${ }^{9}$ At a later stage, it can help encompass the occurrence of traumatising mental events like the recurrence of disturbing thoughts and images, and the prevalence of psychological disorders. This is important because both the behavioural and biological immune systems do not account for mental health, even though psychological stress has been correlated to negative responses of the biological immune system. However, although the combination of cognitive and behavioural influences gives rise to many possibilities, their limits are still not clear. Furthermore, it is unclear how we can go about distinguishing between failures resulting from the cognitive-behavioural immune system and those not related to it. For example, when an individual suffers from chronic psychological disorders, is it because of a dysfunctional psychological immune system or the lack of its engagement? Such issues become difficult to address, especially when performing exercises related to resilience training. ${ }^{4}$ Therefore, there is a need for identifying the factors that result in the engagement of the psychological immune system.

Ultimately, the inclusion of phenomena like resilience, wellbeing, and emotional processing as part of the psychological immune system can help create a unified classification for them. This will help better study the processes that link these phenomena. Additionally, comparing the biological and cognitive-behavioural immune system can bring about op- 
portunities to study the links between the two. For instance, one could track changes in the biological immune system to assess the impact of psychological interventions. Furthermore, the biological phenomena of adaptive immunity could be extremely useful in developing models for resilience in the psychological immune system. Such inquiry is useful because the main questions about resilience involve its appropriate definition, the identification of factors involved in its development, and the possibility of resilience training. ${ }^{3}$

It must be understood that the psychological immune system model does not entirely explain other possible factors that affect psychological immunity. For instance, the social cognitive perspective implies that environmental factors include cultural environments. ${ }^{13}$ Moreover, psychological traits related to immunity can change over time with interactions within the environment. A more complete model for psychological immunity can be developed by analysing an individual's psychological characteristics and familial and peer relationships because it is mediated by social support. ${ }^{13}$

\section{CONCLUSIONS}

The psychological immune system is a construct that comprises 3 subsystems containing over 16 subcomponents. Together, it is a multidimensional construct that offers resistance to psychological trauma and stress. Moreover, psychological immunity is known to have a strong correlation to life expectancy, implying that it is related to phenomena like well-being. The psychological immune system offers a unified framework that can better classify cognitive and behavioural influences; it will be able to better appraise aspects like resilience, which can involve positive behaviour motivated by perceived stress or trauma. Additionally, further development of the psychological immunity construct will allow the investigation and assessment of interactions with the biological immune system, especially in the context of interventions like resilience training. The psychological immune system can encompass resilience, given that many of its characteristics and aspects are similar. This implies that the operationalization of resilience within its framework is possible.

\section{ACKNOWLEDGEMENTS}

Authors acknowledge the immense help received from the scholars whose articles are cited and included in references to this manuscript.

\section{Declaration of Conflicting Interests}

The author(s) declared no potential conflicts of interest with respect to the research authorship, and/or publication of this article.

\section{Source of Funding: Nil}

\section{REFERENCES}

1. Fletcher D, Sarkar M. Psychological resilience: A review and critique of definitions, concepts, and theory. Eur Psychol 2013;18(1):12.

2. Shastri, PC. Resilience: Building immunity in psychiatry. Indian Journal of Psychiatry,2013; 55(3): 224.

3. Truffino JC. Resilience: An approach to the concept. Revista de Psiquiatría y Salud Mental (English Edition), 2010; 3(4), 145151.

4. Rachman SJ. Invited essay: Cognitive influences on the psychological immune system. J Behavior Therap Exp Psychiatry 2013;53, 2-8.

5. Voitkāne S. Goal directedness concerning life satisfaction, psychological immune system and depression in first-semester university students in Latvia. Baltic J Psychol 2004; 5(2):19-30.

6. Pasichnyk I, Handzilevska H, Nikitchuk, U. Psychological immunity of ukrainian migrants depending on childhood scenario sets. Psychol Prosp J, 2017;(30): 145-156.

7. Bonanno GA. Loss, trauma, and human resilience: Have we underestimated the human capacity to thrive after extremely aversive events? Am Psychol 2004;59(1): 20.

8. Choochom O, Sucaromana U, Chavanovanich J, Tellegen P. Model of self-development for enhancing psychological immunity of the elderly. J Behavio Sci 2019;14(1): 84-96.

9. Olah A. Health protective and health-promoting resources in personality: A framework for the measurement of the Psychological Immune System. Positive Psychology Meeting in Budapest, Quality of Life Research Center, Lorand Eōtvōs University, Budapest, Hungary 2000;

10. Bredács AM. Psychological immunity research to the improvement of the professional teacher training's national methodological and training development. Pract Theory Syst Edu 2016;11(2):118-141.

11. Trost K, Demir R. Understanding Psychological Competencies: Conceptualization and Measurement of Psychological Capital at Various Levels. Ratio Working Paper, 2014;251.

12. Oláh A, Nagy H, Tóth KG. Life expectancy and psychological immune competence in different cultures. ETC, 2010;102.

13. Choochom O. Antecedents and consequences of psychological immunity. Humanities Soc Sci Rev, 2014;3(3):191-197. 